•生物编目・

\title{
秦岭中段野生动物多样性的红外相机 监测数据库平台介绍
}

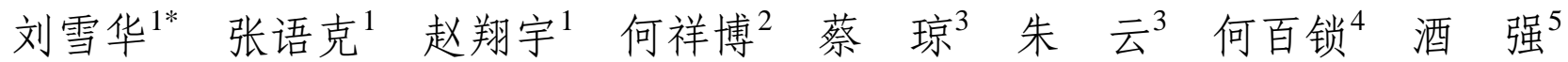

1 (清华大学环境学院, 北京 100084) 2 (佛坪国家级自然保护区, 陕西佛坪 723400)

3 (观音山国家级自然保护区，陕西佛坪 723400)

4 (长青国家级自然保护区, 陕西汉中 723000)

5 (黄柏塬国家级自然保护区，陕西太白 721600)

摘要: 秦岭地处我国中西部, 生物地理位置重要, 拥有丰富的生物多样性, 有大熊猫(Ailuropoda melanoleuca)、秦 岭羚牛(Budorcas bedfordi)、金约㬋 (Rhinopithecus roxellana) 和朱噮(Nipponia nippon)等4个秦岭森林旗舰物种，被称 为“秦岭四宝”。利用红外相机技术开展秦岭野生动物的非损伤性监测不仅可以为秦岭山系提供物种名录信息, 还 可以为了解秦岭野生动物的行为和活动格局提供科学数据。清华大学环境学院生态团队自2009-2020年在秦岭中 段南坡先后实施了7 个项目, 对秦岭南坡的 4 个保护区进行了野生动物监测, 面积达 $1,113 \mathrm{~km}^{2}(26.5 \mathrm{~km} \times 42 \mathrm{~km})$, 红外相机位点数 267 个, 相机日数 152,160 天, 共获取红外相机照片 855,260 张。共鉴定出 27 种野生兽类和 63 种野生 鸟类, 并应用这些照片数据开展了信息挖掘工作, 对野生动物行为、稀有物种、与生境的关系, 以及人为活动对野 生动物的影响等领域进行了研究, 已取得部分成果。在此基础上建立了“秦岭中段野生动物多样性的红外相机监测 数据库平台”, 供团队内部及合作者使用。通过 10 年的监测, 我们提出未来研究建议: (1)对于非常偶见的物种, 还 需要更长的时间并在更多样化的生境布设相机, 以获取更多影像数据评估其现状; (2)数据库需要在更大程度和深 度上进行信息挖掘, 尤其在种间关系、物种-生境关系、种群动态等方面; (3)对典型大种群数量的物种(如秦岭羚牛 和野猪Sus scrofa)及食物链顶端大型捕食动物(如金钱豹Panthera pardus)进行种群动态研究, 为整个秦岭生态系统 的健康持续提供科学支撑; (4)利用数据库的数据及今后红外相机监测数据进行野生动物疾病的发生发展监测研 究。

关键词: 红外相机技术; 野生动物监测; 物种名录; 秦岭中段; 数据库平台

\section{Introduction to the wildlife camera-trapping database of the middle Qinling Mountains}

\author{
Xuehua Liu ${ }^{*}$, Yuke Zhang ${ }^{1}$, Xiangyu Zhao ${ }^{1}$, Xiangbo He ${ }^{2}$, Qiong Cai ${ }^{3}$, Yun Zhu ${ }^{3}$, Baisuo He ${ }^{4}$,iang Jiu ${ }^{5}$ \\ 1 School of Environment, Tsinghua University, Beijing 100084 \\ 2 Foping National Nature Reserve, Foping, Shaanxi 723400 \\ 3 Guanyinshan National Nature Reserve, Foping, Shaanxi 723400 \\ 4 Changqing National Nature Reserve, Hanzhong, Shaanxi 723000 \\ 5 Hangbaiyuan National Nature Reserve, Taibai, Shaanxi 721600
}

\begin{abstract}
The Qinling Mountains, which are located in the midwestern part of China, are biogeographically important because they are home to China's four national wildlife treasures, i.e., giant panda (Ailuropoda melanoleuca), golden takin (Budorcas bedfordi), golden monkey (Rhinopithecus roxellana) and crested ibis (Nipponia nippon). These species are also called the four flagship species in the Qinling forest ecosystem. Therefore, using infrared camera trapping to monitor the wildlife in the Qinling Mountains is very important since it can provide information of species and also scientific data on wildlife behaviour and activity pattern.
\end{abstract}

收稿日期: 2020-04-19; 接受日期: 2020-06-24

基金项目：国家自然科学基金(41271194; 41671183)和原国家林业局大熊猫国际合作专项资金(CM1424, 2017 年项目)

* 通讯作者 Author for correspondence. E-mail: xuehua-hjx@tsinghua.edu.cn 
The ecological research team from Tsinghua University conducted camera trappings between 2009 and 2020 in the Qinling Mountains for seven projects. These projects monitored wildlife diversity, primarily in four nature reserves, covering an area of $1,113 \mathrm{~km}^{2}$ (26.5 km $\left.\times 42 \mathrm{~km}\right)$. There were a total of 267 camera-trapping sites. For the 152,160 camera working days, we were able to obtain totally 855,260 photos. From these photos we identified 27 mammal species and 63 bird species, and were able to address several research aspects including: wildlife behavior, identifying rare species, understanding habitat use and adaption, and understanding the human impacts on wildlife. Based on the gathered photos, we established the wildlife camera-trapping database of the middle Qinling Mountains, which has only been shared within the research group and collaborators. Based on our results from 10 years of monitoring, we propose the following suggestions for future research: (1) We need much longer time and we need to implement camera trapping in more habitat types to collect more digital images to be able to monitor the status of animal species rarely seen in front of cameras. For example, Mustela kathiah, Lutra lutra, and Catopuma temminckii were each captured only once in 10-year monitoring. (2) More deep data mining work is necessary in using this database to understand species-species relationships, species-habitat relationships, and population dynamics. (3) Continual research on the population dynamics of species with large populations (like Budorcas bedfordi and Sus scrofa) and carnivores species at the top of food chain (like Panthera pardus) is able to provide scientific support to the whole Qinling ecosystem. (4) Mining this photo database to monitor and research wildlife disease's occurring as well as developing.

Key words: camera trapping; wildlife monitoring; species list; Qinling; database platform

\section{1 平台简介}

秦岭地处我国中西部, 地理位置重要, 山脉呈 东西走向，拦截了南来的暖湿气流，阻挡了北方的 干冷气流，使得秦岭拥有了丰富的生物多样性，且 滋养了大熊猫(Ailuropoda melanoleuca)、秦岭羚牛 (Budorcas bedfordi)、金丝 猴(Rhinopithecus roxellana) 和朱睘鸟(Nipponia nippon) 4个秦岭森林旗舰物种, 它 们被称为 “秦岭四宝”。利用红外相机技术开展秦岭 野生动物的非损伤性监测不仅可以为秦岭山系提 供物种名录信息, 还可以为了解秦岭野生动物的行 为和活动格局提供科学数据。

秦岭中段大熊猫及其野生动物多样性的红外 相机监测始于 2009 年7月, 到2020年为止实施了监 测时段不等的 7 个项目。数据库建设始于 2009年底, 定名为“秦岭中段野生动物多样性的红外相机监测 数据库平台”, 由清华大学负责建立和维护, 观音 山、佛坪、长青和黄柏塬保护区4家单位参加了监 测和数据收集过程。现在平台仍在建设中, 管理架 构及运行机制也在完善中, 当前还主要供团队内部 及部分合作者使用。

红外相机监测区域仅限于观音山、佛坪、长青 和黄柏塬 4 个保护区, 面积为 $1,113 \mathrm{~km}^{2}$ (26.5 km $\times$ $42 \mathrm{~km})$ 。监测对象为秦岭野生动物。不同时期进入 到数据库平台的项目目标不同, 包括: (1)生境变化
下的动物多样性及其生境利用(观音山保护区); (2) 不同恢复机制下的动物多样性及生境利用(长青、佛 坪和观音山保护区); (3)道路对动物多样性的影响 监测(观音山保护区); (4)大熊猫密度增长区的大熊 猫活动及动物多样性监测(黄柏塬、佛坪和观音山保 护区); (5)秦岭25 ha森林大样地的动物多样性监测; (6)竹林稀疏改造及大熊猫对竹林的应用监测(佛坪 保护区); (7)巴山木竹(Bashania fargesii)恢复种植及 大熊猫利用监测(观音山保护区)。各项目的相机位 点数、起始时间、结束时间、照片数等信息汇总于 表1。

在秦岭开展红外相机监测的单位比较多, 建立 数据库平台的还有北京大学和中国林业科学研究 院, 我们的数据库平台与这两家的数据库平台可能 会在部分区域有重叠。

\section{秦岭中段野生动物的红外相机监测方案}

自2009年7月到现在实施的 7 个红外相机监测 项目，所涉及的监测位点数分别是28、96、20、80、 $30 、 9 、 4$ 个, 共267个(表1), 分布于秦岭中段南坡的 观音山、佛坪、长青和黄柏塬4个自然保护区(图1)。

该数据库平台的红外相机没有按照公里网格 布设, 而是根据不同时期实施的项目目标布设, 均 为单台、常年布设。早期数据是按每隔 $1-2$ 个月进 行一次下载收集, 中后期是按照每3-4个月进行一 
表1 秦岭中段野生动物多样性的红外相机监测数据库平台基本信息表

Table 1 Basic information of the wildlife camera-trapping database platform of the middle Qinling Mountains

\begin{tabular}{|c|c|c|c|c|c|c|c|c|}
\hline $\begin{array}{l}\text { 项目 } \\
\text { Project }\end{array}$ & $\begin{array}{l}\text { 地点 } \\
\text { Location }\end{array}$ & $\begin{array}{l}\text { 保护区 } \\
\text { Nature } \\
\text { reserve }\end{array}$ & $\begin{array}{l}\text { 项目目的 } \\
\text { Aim of project }\end{array}$ & $\begin{array}{l}\text { 起始年月 } \\
\text { Start time }\end{array}$ & $\begin{array}{l}\text { 结束年月 } \\
\text { End time }\end{array}$ & $\begin{array}{l}\text { 有效相机位点 } \\
\text { Effective } \\
\text { camera site }\end{array}$ & $\begin{array}{l}\text { 照片数 } \\
\text { Photos } \\
\left(\times 10^{4}\right)\end{array}$ & $\begin{array}{l}\text { 备注 } \\
\text { Note }\end{array}$ \\
\hline $\begin{array}{l}\text { 按项目需求布设 } \\
\text { Arranged } \\
\text { according to the } \\
\text { needs }\end{array}$ & $\begin{array}{l}\text { 陕西佛坪 } \\
\text { Foping, } \\
\text { Shaanxi }\end{array}$ & $\begin{array}{l}\text { 佛坪、 } \\
\text { 观音山 } \\
\text { Foping, } \\
\text { Guanyin } \\
\text { shan }\end{array}$ & $\begin{array}{l}\text { 监测森林砍伐停止后恢复过程中不 } \\
\text { 同海拔梯度的野生动物多样性 } \\
\text { To monitor wildlife diversity in } \\
\text { different elevation ranges under forest } \\
\text { restoration after stopping logging }\end{array}$ & 2009.7 & 2017.12 & 28 & 23.8708 & \\
\hline $\begin{array}{l}\text { 自然科学基金 } \\
\text { National Natural } \\
\text { Science } \\
\text { Foundation of } \\
\text { China }\end{array}$ & $\begin{array}{l}\text { 陕西佛坪、 } \\
\text { 洋县 } \\
\text { Foping \& } \\
\text { Yangxian, } \\
\text { Shaanxi }\end{array}$ & $\begin{array}{l}\text { 佛坪、 } \\
\text { 观音山、 } \\
\text { 长青 } \\
\text { Foping, } \\
\text { Guanyin } \\
\text { shan, } \\
\text { Changqing }\end{array}$ & $\begin{array}{l}\text { 监测森林砍伐停止后不同恢复机制 } \\
\text { 下的野生动物多样性和生境适应 } \\
\text { To monitor wildlife diversity and } \\
\text { habitat adaptation under different } \\
\text { restoration mechanisms after stopping } \\
\text { logging }\end{array}$ & 2013.7 & 2017.6 & 96 & 30.3511 & $\begin{array}{l}\text { 按项目需求 } \\
\text { 布设 } \\
\text { Arranged } \\
\text { according to } \\
\text { the needs }\end{array}$ \\
\hline $\begin{array}{l}\text { 道路影响监测 } \\
\text { Road impact } \\
\text { monitoring }\end{array}$ & $\begin{array}{l}\text { 陕西佛坪 } \\
\text { Foping, } \\
\text { Shaanxi }\end{array}$ & $\begin{array}{l}\text { 观音山 } \\
\text { Guanyin } \\
\text { shan }\end{array}$ & $\begin{array}{l}\text { 监测保护区中机动车道路对野生动 } \\
\text { 物的影响 } \\
\text { To monitor impact of the road inside } \\
\text { nature reserve on wildlife }\end{array}$ & 2014.7 & 2018.10 & 20 & 17.3689 & $\begin{array}{l}\text { 按项目需求 } \\
\text { 布设 } \\
\text { Arranged } \\
\text { according to } \\
\text { the needs }\end{array}$ \\
\hline $\begin{array}{l}\text { 大熊猫扩散监测 } \\
\text { Dispersal } \\
\text { monitoring of giant } \\
\text { panda }\end{array}$ & $\begin{array}{l}\text { 陕西佛坪、 } \\
\text { 太白 } \\
\text { t Foping \& } \\
\text { Taibai, } \\
\text { Shaanxi }\end{array}$ & $\begin{array}{l}\text { 佛坪、 } \\
\text { 观音山、 } \\
\text { 黄柏塬 } \\
\text { Foping, } \\
\text { Guanyin } \\
\text { shan, } \\
\text { Huangbai } \\
\text { yuan }\end{array}$ & $\begin{array}{l}\text { 监测大熊猫扩散区的大熊猫及其同 } \\
\text { 域物种 } \\
\text { To monitor giant panda and its } \\
\text { sympatric animals in the giant panda } \\
\text { expanding areas }\end{array}$ & 2018.10 & $\begin{array}{l}2019.12 \\
\text { 进行中 } \\
\text { Going on }\end{array}$ & 80 & 8.2155 & $\begin{array}{l}\text { 按项目需求 } \\
\text { 布设 } \\
\text { Arranged } \\
\text { according to } \\
\text { the needs }\end{array}$ \\
\hline $\begin{array}{l}25 \text { ha森林样地监 } \\
\text { 测 } \\
\text { 25ha forest plot } \\
\text { monitoring }\end{array}$ & $\begin{array}{l}\text { 陕西佛坪 } \\
\text { Foping, } \\
\text { Shaanxi }\end{array}$ & $\begin{array}{l}\text { 佛坪 } \\
\text { Foping }\end{array}$ & $\begin{array}{l}\text { 监测25 ha森林大样地的野生动物多 } \\
\text { 样性 } \\
\text { To monitor wildlife diversity and their } \\
\text { small-scale habitat use in a } 25 \text { ha plot }\end{array}$ & 2018.10 & $\begin{array}{l}2019.10 \\
\text { 进行中 } \\
\text { Going on }\end{array}$ & 30 & $\begin{array}{l}1.4869 \\
(2019.12)\end{array}$ & $\begin{array}{l}\text { 按 } 100 \mathrm{~m} \times \\
100 \mathrm{~m} \times \text { 格 } \\
\text { 布设 } \\
\text { Arranged } \\
\text { based on } 100 \\
\mathrm{~m} \times 100 \mathrm{~m} \\
\text { grid }\end{array}$ \\
\hline $\begin{array}{l}\text { 竹子稀疏 } \\
\text { Bamboo thinning }\end{array}$ & $\begin{array}{l}\text { 陕西佛坪 } \\
\text { Foping, } \\
\text { Shaanxi }\end{array}$ & $\begin{array}{l}\text { 佛坪 } \\
\text { Foping }\end{array}$ & $\begin{array}{l}\text { 监测老化竹林稀疏改良后的大熊猫 } \\
\text { 利用情况 } \\
\text { To monitor the giant pandas' use of the } \\
\text { bamboo forest after thinning }\end{array}$ & 2017.6 & 2019.5 & 9 & 2.5474 & $\begin{array}{l}\text { 按项目需求 } \\
\text { 布设 } \\
\text { Arranged } \\
\text { according to } \\
\text { the needs }\end{array}$ \\
\hline $\begin{array}{l}\text { 竹子恢复 } \\
\text { Bamboo } \\
\text { restoration }\end{array}$ & $\begin{array}{l}\text { 陕西佛坪 } \\
\text { Foping, } \\
\text { Shaanxi }\end{array}$ & $\begin{array}{l}\text { 观音山 } \\
\text { Guanyin } \\
\text { shan }\end{array}$ & $\begin{array}{l}\text { 监测大熊猫对恢复种植的巴山木竹 } \\
\text { 林的利用情况 } \\
\text { To monitor the giant panda use of the } \\
\text { re-planted Bashania fargesii forest }\end{array}$ & 2018.12 & 2019.7 & 4 & 1.6854 & $\begin{array}{l}\text { 按项目需求 } \\
\text { 布设 } \\
\text { Arranged } \\
\text { according to } \\
\text { the needs }\end{array}$ \\
\hline
\end{tabular}

覆盖面积达 $1,113 \mathrm{~km}^{2}$, 整个监测覆盖区域的中心位置为 $107.6954^{\circ} \mathrm{E}, 33.6501^{\circ} \mathrm{N}$ 。 The whole area is about $1,113 \mathrm{~km}^{2}$, and its center location is at $107.6954^{\circ} \mathrm{E}, 33.6501^{\circ} \mathrm{N}$.

次下载收集，后期有长达 6 个月 (头年 10 月至来年 3 月)进行收集的, 度过严寒冬季。

\section{3 数据库结构与数据格式}

数据库结构还没有形成一个整体, 仍然是以项 目为基础, 其数据层级为: 项目 -地点-拍摄日期相机号-照片号。数据库管理构架属于分散式, 是以
项目为基础的、非在线的数据系统。数据汇缴方式 也是非在线的。

数据细划分到触发对象类别(兽类、鸟类、自然 干扰、人为干扰、其他), 还包括照片中动物个体数。 根据各个项目的要求, 在数据库中还有环境数据, 如温度、月相，以及拍摄时间等信息。 

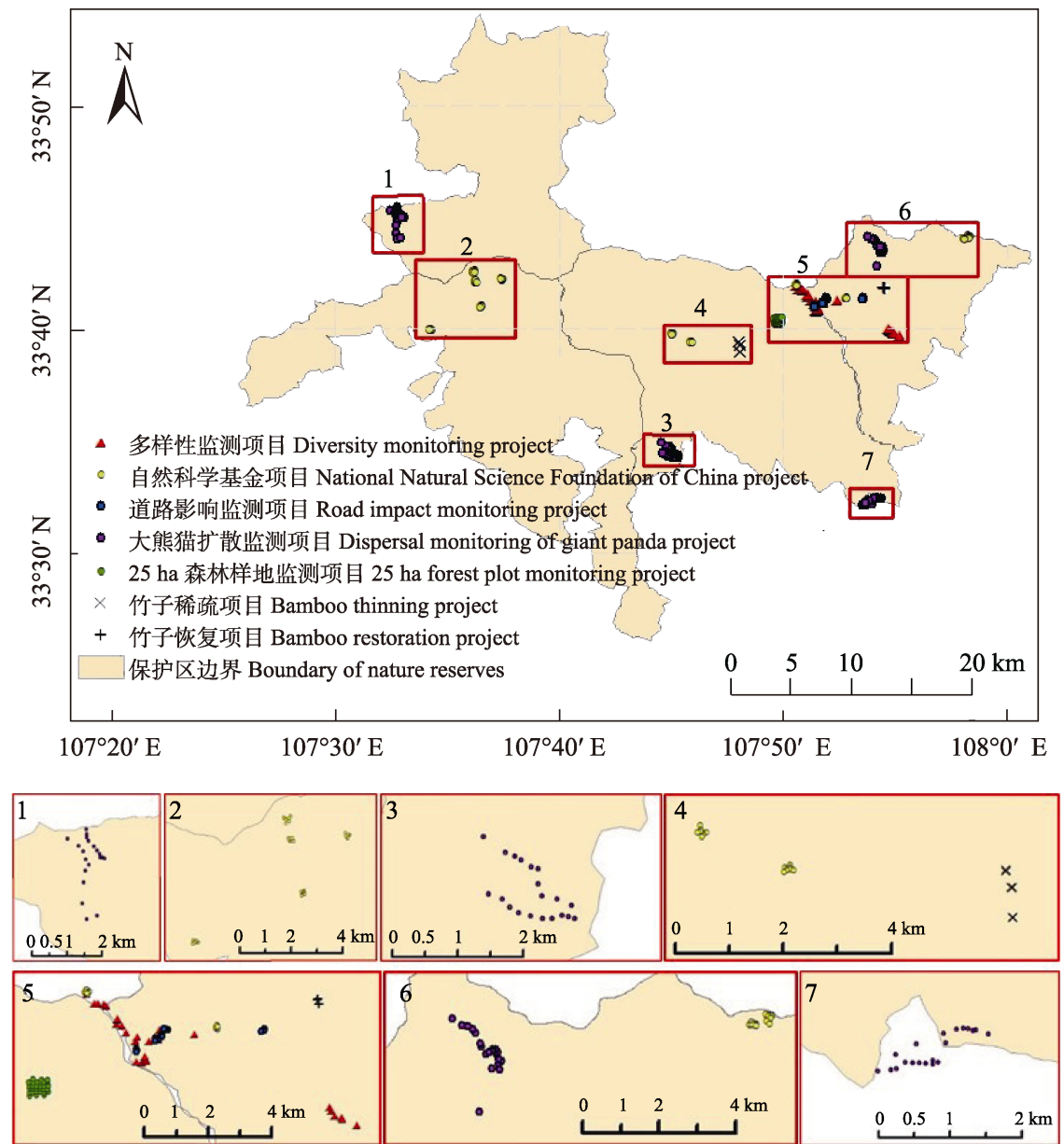

图1 秦岭中段野生动物多样性红外相机监测位点空间分布

Fig. 1 Distribution of camera trapping locations for wildlife monitoring in the middle Qinling Mountains

\section{4 数据量与拍摄物种名录}

至 2019 年 12 月, 总相机工作日数 152,160 d, 照片总数达 855,260 张(已处理的 692,093 张, 待处 理的 163,167 张)。当前仍在野外工作的相机总数 110 台。

兽类分类参考了《中国哺乳动物多样性(第 2 版)》(蒋志刚等, 2017)和《中国脊椎动物红色名录》 (蒋志刚等, 2016), 鸟类分类参考了《中国鸟类分类 与分布名录(第三版)》 (郑光美, 2017)。物种列表是 以项目为基础的, 即每个项目所有监测的红外相机 照片合计。通常, 每个项目的数据库表格字段包括: 相机编号、地点名称、经度、纬度、拍摄日期和具 体时间、月相、温度、类型、物种、个体数等。已 结束项目的数据库都有相对多度指数(relative abundance index, RAI), 是基于各项目收集的数据 计算, 而非所有 7 个项目总数据计算的 RAI。故附
录 1 中物种列表中没有包含 RAI 信息。

基于已经处理的相机数据鉴定到 27 种哺乳动 物和 63 种鸟类(附录1)。27种哺乳动物分属于 5 目 14 科, 包括劳亚食虫目(猬科 1 种)、灵长目 (猴科 1 种)、 啮齿目(松鼠科 4 种、豪猪科 1 种、鼠科 1 种)、食肉目 (大熊猫科1种、熊科1种、鼠科6种、灵猫科1种、猫 科3种)和偶蹄目(猪科 1 种、鹰科 1 种、鹿科 2 种、牛 科3种)。其中, 有国家I级重点保护野生动物 5 种(大 熊猫、川金丝猴、秦岭羚牛、金钱豹Panthera pardus、 林㢌Moschus berezovskii), II级保护兽类6种(黑熊 Ursus thibetanus、中华瓺羚 Capricornis milneedwardsii、中华斑羚Naemorhedus griseus、金 猫Catopuma temminckii、黄喉貂Martes flavigula、 水獭Lutra lutra); 中国特有兽类物种6种(大熊猫、川 金丝猴、秦岭羚牛、小鹿Muntiacus reevesi、侯氏猬 Mesechinus hughi、岩松鼠Sciurotamias davidianus)。 63 种鸟类分属于 8 目 24 科, 包括鸡形目 (雉科 4 
种)、鸽形目(鸠鸽科 1 种)、鸭形目(䂆岛科1种)、鹰形 目(鹰科3种)、鸮形目(鸱鸮科1种)、犀鸟目(戴胜科1 种)、啄木鸟目(豚木鸟科 3 种)、雀形目(伯劳科1种、 鸦科5种、山雀科 3 种、树莺科 1 种、柳莺科 2 种、莺 鹤科 3 种、噪鸬科 6 种、绣眼鸟科 1 种、鸩科 1 种、冻 科 7 种、鹟科 11 种、岩翏科 1 种、雀科 1 种、栺鸰科 1 种、燕雀科2种、鸫科 2 种), 其中雀形目的科和种最 多。鸟类中没有I级保护物种, 但有 8 种II级保护物种, 即勺鸡(Pucrasia macrolopha)、红腹角雉(Tragopan temminckii)、血雉 (Ithaginis cruentus)、红腹锦鸡 (Chrysolophus pictus)、鹰雕(Nisaetus nipalensis)、凤 头鹰(Accipiter trivirgatus)、赤腹鹰(A. soloensis)、北 领角鸮(Otus semitorques); 有中国特有鸟种(郑光美, 2017) 8种，包括红腹锦鸡、黄腹山雀(Pardaliparus venustulus)、黄腹树荀(Horornis acanthizoides)、三 趾鸦雀(Cholornis paradoxus)、斑背噪㓔(Garrulax lunulatus)、橙翅噪啲(G. elliotii)、宝兴歌冻(Turdus mupinensis)、蓝鸤(Emberiza siemsseni)。

\section{重要成果}

在2012-2019年的7年里, 该数据库平台产出如 下重要成果: (1)逐渐完善了秦岭中段区域兽类和鸟 类名录(见附录1), 并对典型物种及特别行为进行了 报道(刘雪华等, 2015, 2018)。(2)开展了多方面的野 生动物行为研究, 包括单物种和类群, 前者如野猪 (王长平等, 2015)和黑熊(成果待发表), 后者如有蹄 类(贾晓东等, 2014); 研究分析了秦岭野生动物的 日活动格局(包括夜行性行为)、季节性活动格局以 及年活动格局(武鹏峰等, 2012; 贾晓东, 2015 ${ }^{(1)}$; 赵 翔宇, $2018^{(2)}$; 许心玉, $2019^{(3)}$ )。(3)研究了野生动物的 生境选择以及在不同森林恢复机制下的生境利用 (林㻦璇, 2016 ${ }^{\oplus}$; 吕子欣, 2016 ${ }^{\circledR}$; Liu et al, 2017; Zhang et al, 2019)。(4)对种群数量大的物种进行了

(1) 贾晓东 (2015) 秦岭南坡不同干扰机制下的有蹄类动物多样性研究. 硕士学位论文, 西北大学, 西安.

(2) 赵翔宇 (2018) 秦岭不同自然生境下野生动物的生境选择与活动规 律研究. 本科学位论文, 清华大学, 北京.

(3) 许心玉 (2019) 秦岭鸟类分布及活动格局的环境影响因子分析. 本 科学位论文, 清华大学, 北京.

(4) 林㻦璇 (2016) 不同干扰机制下秦岭鸟类多样性及活动特征研究. 本科学位论文, 清华大学, 北京.

(5) 吕子欣 (2016) 不同干扰机制下的秦岭兽类多样性及活动特征研究. 本科学位论文, 清华大学, 北京.
种群丰度动态研究(Liu et al, 2013), 提出要关注这 些物种种群的数量变化。(5)分析研究了小空间范围 内物种与物种之间的关系，尤其是在同域生境下的 关系(Zhang et al，待发表)。(6)开展了人为干扰对野 生动物的影响研究, 如森林砍伐的影响(贾晓东, 2015)、道路建设的影响(成果待发表)。(7)开拓了国 际合作，与美国史密森保护生物学会下的保护生态 中心 (Conservation Ecology Center, Smithsonian Conservation Biology Institute)合作开展红外相机监 测秦岭生物多样性, 与韩国江源国立大学开展在红 外相机监测黑熊方面的合作。

\section{6 下一步工作计划及未来建议}

目前, 所有以项目为基础的红外相机数据采集 均以项目的起始和结束时间为监测时间段。本数据 库中仍在进行的两个项目分别是: (1)国家林业与草 原局大熊猫国际合作中心项目一一秦岭大熊猫扩 散的增长-消减区域的非损伤性监测研究，将在 2020 年结束; (2)自筹资金项目一一秦岭 25 ha 森林 大样地的动物多样性及行为监测研究, 将持续到 2023 年(5 年监测); 并计划将大样地的动物多样性 的所有数据上传到中国科学院的生物多样性红外 相机数据库网 (http://cameradata.ioz.ac.cn), 以补充 秦岭森林大样地动物方面的信息。

我们对未来的数据库建设及相关研究提出如 下建议：(1)对于非常偶见的物种, 还需要更长的时 间并在更多样化的生境中布设相机，以获取更多影 像数据以评估其濒危现状及行为活动特征, 如: 本 数据库中 10 年内仅出现 1 次的兽类物种有黄腹鼠、

水獭、金猫; (2)数据库需要在更大程度和深度上进 行信息挖掘, 尤其在种间关系、物种-生境关系、种 群动态等方面; (3)对典型大种群数量的物种(如羚 牛和野猪)及食物链顶端大型捕食动物(如金钱豹) 进行种群动态研究, 为整个秦岭生态系统的健康持 续提供科学支撑; (4)利用数据库的数据及今后红外 相机监测数据进行野生动物疾病的发生发展监测 研究。

\section{参考文献}

Jia XD, Liu XH, Yang XZ, Wu PF, Songer M, Cai Q, He XB, Zhu Y (2014) Seasonal activity patterns of ungulates in Qinling Mountains based on camera-trap data. Biodiversity 
Science, 22, 737-745. (in Chinese with English abstract) [贾 晓东, 刘雪华, 杨兴中, 武鹏峰, Melissa Songer, 蔡琼, 何祥博, 朱云 (2014) 利用红外相机分析秦岭有蹄类动 物多样性及活动规律. 生物多样性, 22, 737-745.]

Jiang ZG, Jiang JP, Wang YZ, Zhang E, Zhang YY, Li LL, Xie F, Cai B, Cao L, Zheng GM, Dong L, Zhang ZW, Ding P, Luo ZH, Ding CQ, Ma ZJ, Tang SH, Cao WX, Li CW, Hu HJ, Ma Y, Wu Y, Wang YX, Zhou KY, Liu SY, Chen YY, Li JT, Feng ZJ, Wang Y, Wang B, Li C, Song XL, Cai L, Zang CX, Zeng Y, Meng ZB, Fang HX, Ping XG (2016) Red List of China's Vertebrates. Biodiversity Science, 24, 500-551. (in Chinese and in English) [蒋志刚, 江建平, 王 跃招, 张鹗, 张雁云, 李立立, 谢锋, 蔡波, 曹亮, 郑光 美, 董路, 张正旺, 丁平, 罗振华, 丁长青, 马志军, 汤宋 华, 曹文宣, 李春旺, 胡慧建, 马勇, 吴毅, 王应祥, 周开 亚, 刘少英, 陈跃英, 李家堂, 冯祚建, 王燕, 王斌, 李 成, 宋雪琳, 蔡蕾, 蔵春金金, 曾岩, 孟智斌, 方红霞, 平晓 鸽 (2016) 中国脊椎动物红色名录. 生物多样性, 24, 500-551.]

Jiang ZG, Liu SY, Wu Y, Jiang XL, Zhou KY (2017) China's mammal diversity (2nd edition). Biodiversity Science, 25, 886-895. (in Chinese with English abstract) [蒋志刚, 刘少 英, 吴毅, 蒋学龙, 周开亚 (2017) 中国哺乳动物多样性 (第2版). 生物多样性, 25, 886-895.]

Liu XH, Cai Q, Jia XD, Wu PF, Cheng HF (2015) Animal world living in Foping of the southern slope of the Qinling Mountains. Forest and Human, (3), 10513. (in Chinese) [刘雪华, 蔡琼, 贾晓东, 武鹏峰, 成鸿飞 (2015) 生活在 佛坪-秦岭南坡的动物世界. 森林与人类, (3), 105-113.]

Liu XH, Wu PF, Cai Q, He XB, Songer M, Zhu Y, Shao XM (2013) Monitoring wildlife abundance and diversity with infra-red camera traps in Guanyinshan Nature Reserve of Shaanxi Province, China. Ecological Indicators, 33(10), 121-128.

Liu XH, Wu PF, He XB, Zhao XY (2018) Application and data mining of infra-red camera in the monitoring of species. Biodiversity Science, 26, 850-861. (in Chinese with English abstract) [刘雪华, 武鹏峰, 何祥博, 赵翔宇 (2018) 红外 相机技术在物种监测中的应用及数据挖掘. 生物多样性, 26, 850-861.]
Liu XH, Wu PF, Shao XM, Songer M, Cai Q, He XB, Zhu Y (2017) Diversity and activity patterns of sympatric animals among four types of forest habitat in Guanyinshan Nature Reserve in the Qinling Mountains, China. Environmental Science and Pollution Research, 24, 16465-16477.

Wang CP, Liu XH, Wu PF, Shao XM (2015) Research on behavior and abundance of wild boar (Sus scrofa) via infrared camera in Guanyinshan Nature Reserve in Qinling Mountains, China. Acta Theriologica Sinica, 35, 14756. (in Chinese with English abstract) [王长平, 刘雪华, 武鹏 峰, 邵小明 (2015) 应用红外相机技术研究秦岭观音山 自然保护区内野猪的行为和丰富度. 兽类学报, 35, 147-156.]

Wu PF (2013) Giant Panda Habitat Assessment and Sympatric Animal Diversity Analysis in Guanyinshan Nature Reserve, Shaanxi. China Agricultural University, PhD dissertation, China Agricultural University, Beijing. (in Chinese with English abstract) [武鹏峰 (2013) 陕西观音山自然保护区 大熊猫栖息地评价及伴生动物多样性分析. 博士学位论 文, 中国农业大学, 北京.]

Wu PF, Liu XH, Cai Q, He XB, Songer M, Zhu Y, Shao XM (2012) The application of infrared camera in mammal research in Guanyinshan Nature Reserve, Shaanxi. Acta Theriologica Sinica, 32, 6771. (in Chinese with English abstract) [武鹏峰, 刘雪华, 蔡琼, 何祥博, Melissa Songer, 朱云, 邵小明 (2012) 红外相机技术在陕西观音山自然 保护区兽类监测研究中的应用. 兽类学报, 32, 67-71.]

Zhang YK, He XB, Liu XH (under review) Fine-scale activity patterns of large-and-medium mammals in deciduous broadleaf forest. Journal of Forest Research. (in press)

Zhang YK, Liu XH, Zhao XY, Lv ZX (2019) Animal diversity responding to different forest restoration schemes in the Qinling Mountains, China. Ecological Engineering, 136, 23-29.

Zheng GM (2017) China Bird Classification and Distribution List, 3rd edn. Science Press, Beijing. (in Chinese) [郑光美 (2017) 中国鸟类分类与分布名录(第三版). 科学出版社, 北京.]

(责任编委: 蒋学龙 责任编辑: 时意专)

\section{附录 Supplementary Material}

附录1 秦岭中段野生动物多样性的红外相机监测数据库平台记录的野生动物物种名录(截至2019年12月)

Appendix 1 Species list of the wildlife camera-trapping database of the middle Qinling Mountains (Before December 2019) http://www.biodiversity-science.net/fileup/PDF/2020094-1.pdf 
刘雪华, 张语克, 赵翔宇, 何祥博, 蔡琼, 朱云, 何百锁, 酒强 (2020) 秦岭中段野生动物多样性的红外相机监测数据库平台介绍. 生物多样 性, 28 (9): 1075-1080.

http://www.biodiversity-science.net/CN/10.17520/biods.2020094

附录1 秦岭中段野生动物多样性的红外相机监测数据库平台记录的野生动物物种名录（截至2019年12月）。“ $\Delta$ ”指中国特 有种。CR, EN, VU, NT和LC分别为极危, 濒危, 易危, 近危和无危。

Appendix 1 Species list of the wildlife camera-trapping database of the middle Qinling Mountains (Before December 2019).

$\Delta$ Chinese endemic species. CR, Critically endangered; EN, Endangered; VU, Vulnerable; NT, Near threatened; LC, Least concern.

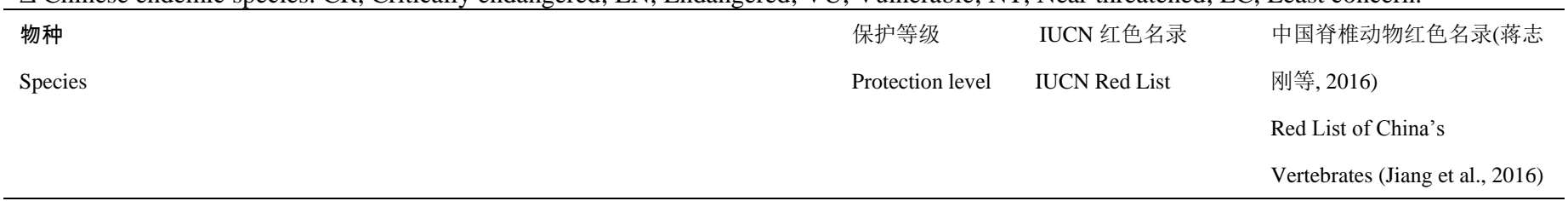

\section{兽类 Mammals}

(一)劳亚食虫目 Eulipotyphla

(1)猬科 Erinaceidae

1. 侯氏猬 Mesechinushughis

$\mathrm{LC}$

NT

(二)灵长目 Primates

(2)猴科 Ceropithecidae

2. 川金丝猴 Rhinopithecusroxellanas

\section{(三)啮齿目 Rodentia}

(3)松鼠科 Sciuridae
3. 岩松鼠 Sciurotamiasdavidianus $\Delta$
LC
LC
4. 珀氏长吻松鼠 Dremomyspernyi
LC
LC
5. 赤腹松鼠 Gallosiuruseythraeus
LC
LC
6. 隐纹花松鼠 Tamiopsswinhoei
LC
LC

(4)豪猪科 Hystricidae

7. 中国豪猪 Hystrixhodgsoni

(5)鼠科 Muridae

8. 北社鼠 Niviventerconfucianus

LC

LC

\section{(四)食肉目 Carnivora}

(6)大熊猫科 Ailuropodidae

9. 大熊猫 Ailuropodamelanoleuca $\Delta$

(7)熊科 Ursidae

$$
\text { 10. 黑熊 Ursusthibetanus }
$$

(8)鼣科 Mustelidae
11. 黄鼠 Mustelasibirica
12. 黄腹鼣 Mustelakathiah
13. 猪獾 Arctonyxcollaris
14. 亚洲狗獾 Melesleucurus
15. 黄喉貂 Martesflavigula
16. 水獭 Lutralutra

(9)灵猫科 Viverridae

17. 果子狸 Pagumalarvata 
刘雪华, 张语克, 赵翔宇, 何祥博, 蔡琼, 朱云, 何百锁, 酒强 (2020) 秦岭中段野生动物多样性的红外相机监测数据库平台介绍. 生物多样 性, 28 (9): 1075-1080.

http://www.biodiversity-science.net/CN/10.17520/biods.2020094

\begin{tabular}{|c|c|c|c|}
\hline 物种 & 保护等级 & IUCN 红色名录 & 中国脊椎动物红色名录(蒋志 \\
\hline \multirow{2}{*}{ Species } & & & Red List of China's \\
\hline & & & Vertebrates (Jiang et al., 2016) \\
\hline 19. 豹猫 Prionailurusbengalensis & & LC & VU \\
\hline \multicolumn{4}{|l|}{ (11)猪科 Suidae } \\
\hline 21. 野猪 Susscrofa & & LC & $\mathrm{LC}$ \\
\hline \multicolumn{4}{|l|}{ (12)瘪科 Moschidae } \\
\hline 22. 林鹿 Moschusberezovskii & I & EN & $\mathrm{CR}$ \\
\hline 24. 小鹿 Muntiacusreevesis & & $\mathrm{LC}$ & VU \\
\hline \multicolumn{4}{|l|}{ (14)牛科 Bovidae } \\
\hline 25. 秦岭羚牛 Budorcasbedfordis & I & & VU \\
\hline 26. 中华鬛羚 Capricornismilneedwardsii & II & NT & VU \\
\hline 27. 中华斑羚 Naemorhedusgriseus & II & VU & VU \\
\hline
\end{tabular}

兽类总计 Mammal total: 27 种

\section{鸟类 Birds}

\section{(一)鸡形目 Galliformes}

(1)雉科 Phasianidae

1. 勺鸡 Pucrasiamacrolopha

2. 红腹角雉 Tragopantemminckii

3. 血雉 Ithaginiscruentus

LC

4. 红腹锦鸡 Chrysolophuspictus $\Delta$

(二)鸽形目 Columbiformes

(2)鸠鸽科 Columbidae

5. 山斑鸠 Streptopeliaorientalis

\section{(三)微形目 Charadriiformes}

(3)鹬科 Scolopacidae

6. 丘㛚 Scolopaxrusticola

\section{(四)鹰形目 Accipitriformes}

(4)鹰科 Accipitridae

7. 鹰雕 Nisaetusnipalensis

8. 凤头鹰 Accipiter trivirgatus

9. 赤腹鹰 Accipiter soloensis

(五)鸮形目 Strigiformes

(5)鸱鸮科 Strigidae 
刘雪华, 张语克, 赵翔宇, 何祥博, 蔡琼, 朱云, 何百锁, 酒强 (2020) 秦岭中段野生动物多样性的红外相机监测数据库平台介绍. 生物多样 性, 28 (9): 1075-1080.

http://www.biodiversity-science.net/CN/10.17520/biods.2020094

\begin{tabular}{|c|c|c|c|}
\hline 物种 & 保护等级 & IUCN 红色名录 & 中国脊椎动物红色名录(蒋志 \\
\hline \multirow{2}{*}{ Species } & & & Red List of China's \\
\hline & & & Vertebrates (Jiang et al., 2016) \\
\hline
\end{tabular}

(六)犀鸟目 Bucerotiformes

(6)戴胜科 Upupidae

11. 戴胜 Upupaepops

LC

LC

(七)啄木鸟目 Piciformes

(7)豚木鸟科 Picidae
12. 灰头绿豚木鸟Picuscanus
13. 大斑啄木鸟 Dendrocopos major
14. 白背啄木鸟 Dendrocoposleucotos

LC

LC

LC

LC

LC

LC

\section{(八)雀形目 Passeriformes}

(8)伯劳科 Laniidae

15. 红尾伯劳 Laniuscristatus

LC

LC

(9)鸦科 Corvidae
16. 星鸦 Nucifragacaryocatactes
17. 大嘴乌鸦 Corvusmacrorhynchos
18. 松鸦 Garrulusglandarius
19. 喜鹊 Pica pica
20. 红嘴蓝鹊 Urocissaerythrorhyncha
(10)山雀科 Paridae

LC

LC

LC

LC

LC

LC

LC

LC

LC

LC

21. 黄腹山雀 Pardaliparusvenustulus $\Delta$

LC

LC

22. 绿背山雀 Parusmonticolus

LC

LC

23. 黑冠山雀 Periparusrubidiventris

LC

LC

(11)树莺科 Cettiidae

24. 黄腹树莺 Horornisacanthizoides $\Delta$

(12)柳莺科 Phylloscopidae

25. 华西柳莺 Phylloscopusoccisinensis

26. 鹃莺属 1 种 Seicercus sp.

(13)莺鸤科 Sylviidae

27. 红嘴鸦雀 Conostomaaemodium

28. 棕头鸦雀 Sinosuthorawebbiana

LC

LC

29. 三趾鸦雀 Cholornisparadoxus $\Delta$

NT

NT

(14)噪鿬科 Leiothrichidae

30. 白喉噪鸤 Garrulaxalbogularis

31. 斑背噪帕 Garrulaxlunulatus $\Delta$

LC

LC

32. 黑领噪鸬 Garrulaxpectoralis

LC

LC

33. 灰翅噪鹛 Garrulaxcineraceus

LC

LC

34. 橙翅噪䴗 Trochalopteronelliotii $\Delta$

LC

LC

35. 红嘴相思鸟 Leiothrixlutea

LC

NT 
刘雪华, 张语克, 赵翔宇, 何祥博, 蔡琼, 朱云, 何百锁, 酒强 (2020) 秦岭中段野生动物多样性的红外相机监测数据库平台介绍. 生物多样 性, 28 (9): 1075-1080.

http://www.biodiversity-science.net/CN/10.17520/biods.2020094

\begin{tabular}{|c|c|c|c|}
\hline 物种 & 保护等级 & IUCN 红色名录 & 中国脊椎动物红色名录(蒋志 \\
\hline \multirow[t]{3}{*}{ Species } & Protection level & IUCN Red List & 刚等, 2016) \\
\hline & & & Red List of China's \\
\hline & & & Vertebrates (Jiang et al., 2016) \\
\hline
\end{tabular}

(15)绣眼鸟科 Zosteropidae

36. 白领凤鹋 Yuhinadiademata

(16)鳞胸鹪鹋科 Pnoepygidae

37. 小鳞胸鹪鹛 Pnoepygapusilla

(17)鸩科 Sittidae

38. 普通鸩Sittaeuropaea

(18)冻科 Turdidae
39. 宝兴歌鸫 Turdusmupinensis $\Delta$
40. 灰头轪 Turdusrubrocanus
41. 灰翅斩 Turdusboulboul
42. 乌鸫 Turdusmandarinus
43. 虎斑地鸫 Zootheraaurea
44. 淡背地鸫 Zootheramollissima
45. 长尾地鸫 Zootheradixoni

LC

LC

LC

LC

LC

LC

LC

LC

LC

LC

LC

LC

LC

LC

(19)能科 Muscicapidae

46. 红腹红尾鸲 Phoenicuruserythrogastrus

LC

47. 黑喉红尾鸲 Phoenicurushodgsoni

LC

LC

48. 紫啸鸫 Myophonuscaeruleus

LC

LC

49. 北红尾鸲 Phoenicurusauroreus

LC

LC

50. 棕腹大仙暡 Niltavadavidi

LC

LC

51. 棕腹仙鹟 Niltavasundara

LC

LC

52. 白顶溪鸲 Chaimarrornisleucocephalus

LC

LC

53. 蓝眉林鸲 Tarsigerrufilatus

LC

LC

54. 金色林鸲Tarsigerchrysaeus

LC

LC

55. 白眉林鸲 Tarsigerindicus

LC

LC

56. 灰蓝姬鹃 Ficedula tricolor

LC

LC

(20)岩鹨科 Prunellidae

57. 棕胸岩翏 Prunellastrophiata

(21)雀科 Passeridae

58. 灰头灰雀 Pyrrhulaerythaca

(22)能鸰科 Motacillidae

59. 田鹨 Anthusrichardi

(23)燕雀科 Fringillidae

60. 酒红朱雀 Carpodacusvinaceus

61. 黑头蜡嘴雀 Eophonapersonata

LC

LC

(24)鸥科 Emberizidae

62. 小鸥 Emberizapusilla 
刘雪华, 张语克, 赵翔宇, 何祥博, 蔡琼, 朱云, 何百锁, 酒强 (2020) 秦岭中段野生动物多样性的红外相机监测数据库平台介绍. 生物多样 性, 28 (9): 1075-1080.

http://www.biodiversity-science.net/CN/10.17520/biods.2020094

\begin{tabular}{|c|c|c|c|}
\hline 物种 & 保护等级 & IUCN 红色名录 & 中国脊椎动物红色名录(蒋志 \\
\hline \multirow[t]{3}{*}{ Species } & Protection level & IUCN Red List & 刚等, 2016) \\
\hline & & & Red List of China's \\
\hline & & & Vertebrates (Jiang et al., 2016) \\
\hline 63. 蓝鸤 Emberizasiemsseni $\Delta$ & & $\mathrm{LC}$ & $\mathrm{LC}$ \\
\hline
\end{tabular}


刘雪华, 张语克, 赵翔宇, 何祥博, 蔡琼, 朱云, 何百锁, 酒强 (2020) 秦岭中段野生动物多样性的红外相机监测数据库 平台介绍. 生物多样性, 28 (9): 1075-1080.

http://www.biodiversity-science.net/CN/10.17520/biods.2020094 